\title{
Otimização da permeabilidade de filtros de aerossóis para altas temperaturas preparados a partir da técnica de adição de espuma aquosa em suspensão cerâmica
}

\author{
(Permeability optimization of hot aerosol filters prepared from \\ foaming of ceramic suspensions)
}

\author{
M. D. M. Innocentini ${ }^{1}$, R. C. O. Romano ${ }^{2}$, H. Schreurs ${ }^{2}$, V. P. Rodrigues ${ }^{1}$, J. R. Coury $^{3}$, R. G. Pileggi ${ }^{2}$ \\ ${ }^{1}$ Engenharia Química, Universidade de Ribeirão Preto - UNAERP \\ ${ }^{2}$ Engenharia Civil, Universidade de S. Paulo - USP \\ ${ }^{3}$ Engenharia Química, Universidade Federal de S. Carlos - UFSCar \\ rcorjau@gmail.com,rafael.pileggi@poli.usp.br, muriloinnocentini@yahoo.com.br
}

\begin{abstract}
Resumo
Este trabalho é parte de um projeto de desenvolvimento de filtros cerâmicos para a remoção de material particulado disperso em correntes gasosas em temperaturas elevadas. Os filtros cerâmicos utilizados para esta finalidade devem apresentar elevada porosidade aliada a uma distribuição homogênea de poros interconectados, resultando em estrutura permeável e com boa resistência mecânica e eficiência de coleta. Uma rota de processamento recentemente desenvolvida é utilizada para o processamento das peças, já que permite a obtenção de todas as características requeridas para uma filtração eficiente. Nesta técnica, os materiais porosos são processados a partir da incorporação de espumas aquosas em uma suspensão cerâmica de alumina e consolidados a partir da adição de cimento aluminoso. Comparado com outras rotas de processamento, este processo é mais atrativo, pois permite a fabricação de peças com geometrias complexas, economicamente viáveis e sem utilização de aditivos tóxicos. O objetivo deste trabalho foi otimizar a composição cerâmica de modo a atender aos requisitos de permeabilidade do filtro, tendo como base sua operação econômica em temperaturas elevadas em processos como incineração de resíduos, produção de cimento ou queima de biomassa em caldeiras em plantas químicas.

Palavras-chave: cerâmica porosa, permeabilidade, filtros cerâmicos, aerossóis, limpeza de gases.
\end{abstract}

\begin{abstract}
This work is part of a Brazilian project to develop ceramic filters to treat flue gases from cement plants, biomass boilers and waste incinerators. Ceramic filters used for these purposes must present high porosity, homogeneous porous distribution (interconnected) to result in bodies with high mechanical strength, permeability and collection efficiency for fine particles. A method recently developed has been used for the filters processing. In this new processing route, the porous samples were prepared through the incorporation of aqueous foams into alumina-based suspensions. Compared to other techniques, this process seems to be an attractive and cost effective solution for fabricating complex shaped bodies, without toxic admixtures. The procedure to establish the required features of the filtering membrane for a desired flow condition is described, as well as the experimental work to produce and select the ceramic membranes with such requirements.
\end{abstract}

Keywords: porous ceramics, permeability, ceramic filters, flue gases, aerosol, gas cleaning.

\section{INTRODUÇ̃̃O}

Cerâmicas porosas celulares combinam propriedades estruturais, mecânicas e fluidodinâmicas que favorecem o uso na filtração de partículas em correntes gasosas a altas temperaturas [1-4]. Para o tratamento bem sucedido de emissões atmosféricas de aerossóis provenientes de fornos de fabricação de cimento, refinarias, caldeiras e motores à combustão, os filtros cerâmicos devem ter sua permeabilidade maximizada, de modo que a queda de pressão através do elemento seja pequena e implique em baixo consumo energético. Esse requisito pode ser atingido durante o processamento cerâmico adequando-se variáveis como morfologia, tamanho e volume de poros. No entanto, muita atenção deve ser dada às variáveis interdependentes, como a resistência mecânica e a eficiência de coleta de partículas, para assegurar o desempenho solicitado por uma longa vida útil.

A fim de se conseguir maior controle sobre as características da membrana filtrante, é muito importante selecionar a técnica de processamento adequada para obtenção da estrutura porosa desejada [5-6]. Dentre as 
técnicas de processamento de cerâmicas porosas existentes, destacam-se o método de incorporação e queima de partículas orgânicas, o método da réplica de esponjas poliméricas, o método de gelcasting e a técnica de incorporação de espuma aquosa em suspensão cerâmica [5-6]. Nas três primeiras técnicas, as cerâmicas obtidas não apresentam homogeneidade microestrutural, a faixa de diâmetros de poros é ampla e dificilmente se tem o controle da porosidade gerada, sendo difícil a reprodução de peças com as mesmas características, em diferentes bateladas. Além disso, no caso do gelcasting, muitas vezes são utilizados aditivos tóxicos e algumas vezes cancerígenos. Desta forma, visando obter cerâmicas com as características desejadas para utilização em filtros de aerossóis, os materiais podem ser processados a partir da técnica de incorporação de espumas aquosas em uma suspensão de alumina [5], na qual tanto a porosidade quanto a distribuição dos poros podem ser controladas, e as peças são obtidas com considerável resistência mecânica, homogeneidade estrutural e alta permeabilidade.

\section{Otimização de permeabilidade}

A permeabilidade é uma medida macroscópica que determina a facilidade com que um fluido submetido a um gradiente de pressão percola os vazios em um meio poroso. Uma adequada descrição de permeabilidade deve, portanto, combinar aspectos do fluido, da estrutura porosa e do escoamento. A equação de Forchheimer é um exemplo de uma boa relação aceita na literatura, sendo expressa por uma relação parabólica entre a variação de pressão através do meio $\Delta \mathrm{P}$ e a velocidade superficial $\mathrm{v}_{\mathrm{s}}[2,6-10]$ :

$$
\frac{\Delta \mathrm{P}}{\mathrm{L}}=\frac{\mu}{\mathrm{k}_{1}} \mathrm{v}_{\mathrm{s}}+\frac{\rho}{\mathrm{k}_{2}} \mathrm{v}_{\mathrm{s}}^{2}
$$

Para fluidos incompressíveis (líquidos):

$$
\Delta \mathrm{P}=\mathrm{P}_{\mathrm{e}}-\mathrm{P}_{\mathrm{s}}
$$

e para fluidos compressíveis (gases e vapores):

$$
\Delta \mathrm{P}=\frac{\mathrm{P}_{\mathrm{e}}^{2}-\mathrm{P}_{\mathrm{s}}^{2}}{2 \mathrm{P}}
$$

Nas equações (A-C), $\mathrm{P}_{\mathrm{e}}$ e $\mathrm{P}_{\mathrm{s}}$ são, respectivamente, as pressões absolutas na entrada e na saída da membrana, $\mathrm{P}$ é a pressão do fluido para a qual a velocidade $\mathrm{v}_{\mathrm{s}}$ é medida e a viscosidade $(\mu)$ e a densidade $(\rho)$ fluido são calculadas.

Para as condições típicas de filtração em altas temperaturas, a queda de pressão através do elemento filtrante é baixa comparada à pressão da corrente gasosa, e deste modo o erro associado ao uso da Equação (B) é menor que $2 \%$.

Para meios filtrantes rígidos, tais como as cerâmicas porosas, acontribuiçãodaestruturanaequaçãoAéquantificada através da espessura $\mathrm{L}$ e dos parâmetros de permeabilidade $\mathrm{k}_{1}$ e $\mathrm{k}_{2}$, que dependem de modo complexo da morfologia e da distribuição de tamanho de poros, porosidade, conectividade dos poros e da porosidade. Usualmente, os parâmetros $\mathrm{k}_{1}$ e $\mathrm{k}_{2}$ são referidos como constantes de permeabilidade, uma vez que eles são supostamente independentes do fluido permeante ou das condições de escoamento, mesmo embora eles possam variar com a temperatura $[4,11]$. Os termos $\mathrm{k}_{1} \mathrm{e}$ $\mathrm{k}_{2}$ são assim conhecidos como constantes de permeabilidade Darciana e não-Darciana, em referência à lei de Darcy, um modelo empírico simplificado para a descrição de permeabilidade em meios porosos.

A equação A é útil para quantificar a energia (pressão) necessária para forçar o gás percolar a membrana filtrante com a velocidade de filtração $\mathrm{v}_{\mathrm{s}}$ desejada. Os maiores custos relacionados à operação de um filtro para temperaturas elevadas estão relacionados à área de filtração (que implica no número de unidades ou elementos de filtração), à queda de pressão (que implica na potência elétrica do ventilador) e à vida útil do elemento filtrante (que implica na freqüência de aquisição e troca dos filtros).

$\mathrm{O}$ consumo elétrico é diretamente proporcional à queda de pressão $\Delta \mathrm{P}$ e à vazão volumétrica dos gases $\mathrm{Q}$ :

$$
\mathrm{W}=\frac{\mathrm{Q} \Delta \mathrm{P}}{\eta}
$$

sendo $\eta$ o rendimento do ventilador (geralmente entre 50 e $80 \%)$.

Tipicamente, Q é definido pela aplicação e a área necessária de filtração A é então calculada por $\mathrm{A}=\mathrm{Q} / \mathrm{v}_{\mathrm{s}}$ para resultar em queda de pressão e consumo elétrico aceitáveis. Assim, o número de elementos filtrantes $\mathrm{n}$ é obtido dividindo-se a área total de filtração pela área de cada elemento $\left(n=A / A_{i}\right)$.

Do ponto de vista industrial, a filtração de aerossóis em altas temperaturas opera com velocidades superficiais entre 1 e $10 \mathrm{~cm} / \mathrm{s}$, com quedas de pressão aceitáveis para o meio virgem menores do que $1000 \mathrm{~Pa}\left(\sim 10 \mathrm{cmH}_{2} \mathrm{O}\right)$ [14]. Velocidades maiores que $10 \mathrm{~cm} / \mathrm{s}$ são desejáveis, mas elas podem implicar no aumento excessivo na queda de pressão, além de poder causar a penetração de partículas na estrutura, com o progressivo entupimento dos poros e diminuição da vida útil do elemento filtrante. Por outro lado, a diminuição da velocidade de filtração para valores menores que $1 \mathrm{~cm} / \mathrm{s}$ implica em baixa queda de pressão e consumo energético, mas por outro lado a área de filtração é aumentada proporcionalmente, assim como os custos de aquisição e manutenção dos elementos filtrantes.

Uma vez que a dependência entre a queda de pressão e a velocidade do fluido é parabólica, conforme a equação A, então para dobrar a velocidade do gás através da membrana é necessário que a queda de pressão seja aumentada entre 2 e 4 vezes, com aumento proporcional na potência consumida pelo ventilador. Pela análise da equação A, as alternativas para minimizar a queda de pressão e assim os custos devidos às restrições de área de filtração são então a diminuição da espessura da membrana $\mathrm{L}$ ou o aumento das constantes de permeabilidade $\mathrm{k}_{1}$ e $\mathrm{k}_{2}$. A primeira opção é facilmente obtida durante o processamento cerâmico, promove redução linear 
de queda de pressão e não afeta a eficiência de coleta de partículas do aerossol, mas por outro lado pode comprometer a resistência mecânica da membrana às solicitações de filtração e assim reduzir a vida útil do filtro.

Uma redução na queda de pressão também pode ser conseguida pelo aumento das constantes de permeabilidade $\mathrm{k}_{1}$ e $\mathrm{k}_{2}$, sendo que a intensidade dessa redução irá depender do peso de cada termo do lado direito da equação de Forchheimer. O termo linear $\left[\mu v_{s} / k_{1}\right]$ representa a perda de energia por dissipação viscosa entre as camadas de fluido e prevalece para número de Reynolds no poro menor que 1 $\left(\operatorname{Re}_{\text {poro }}=\rho v_{\text {s }} \mathrm{d}_{\text {poro }} / \varepsilon \mu<1\right)$, sendo $\mathrm{d}_{\text {poro }}$ o diâmetro médio de poro e $\varepsilon$ a porosidade da estrutura. O termo quadrático $\left[\rho_{\mathrm{s}}{ }_{\mathrm{s}} / \mathrm{k}_{2}\right]$, por sua vez, representa a perda de pressão por ação inercial, causada pela alternância de aceleração e desaceleração do fluido e por sua mudança de trajetória durante a permeação pelos poros. Este termo é predominante para 1-10 $<\mathrm{Re}_{\text {por }}$ $<150$ e ocorre dentro do regime de escoamento laminar. E importante destacar que o termo inercial não representa o início da turbulência propriamente dita no escoamento do fluido, o que geralmente ocorre $\mathrm{Re}_{\text {poro }}>300$ [12].

Dada a dificuldade em quantificar em escala microscópica os parâmetros para a análise do regime de escoamento em meios porosos, é comum expressar a equação A em termos do número de Forchheimer Fo [2]:

$$
\begin{aligned}
& \mathrm{Fo}_{\mathrm{O}}=\frac{\rho \mathrm{v}_{\mathrm{s}}\left(\mathrm{k}_{1} / \mathrm{k}_{2}\right)}{\mu} \\
& \frac{\Delta \mathrm{P}}{\mathrm{L}}=\frac{\mu}{\mathrm{k}_{1}} \mathrm{v}_{\mathrm{s}}(1+\mathrm{Fo})
\end{aligned}
$$

Uma vez que a razão $\mathrm{k}_{1} / \mathrm{k}_{2}$ tem dimensão de comprimento, o parâmetro Fo na equação E pode ser entendido como um análogo macroscópico do número de Reynolds, estando relacionado à linearidade da equação A. Assim, para minimizar os efeitos inerciais sobre a queda de pressão, o parâmetro Fo deve ser pequeno ( $\mathrm{Fo}<<1)$, quando então a equação A é reduzida à lei de Darcy.

É importante destacar que não é a velocidade do fluido $v_{\mathrm{s}}$ em si que determina a linearidade da equação de Forchheimer, mas sim o $\mathrm{Re}_{\text {poro }}$ em escala microscópica ou o parâmetro Fo em escala macroscópica (Para Fo = 1, $50 \%$ da perda de pressão no meio poroso é devida ao termo viscoso e $50 \%$ ao termo inercial). Para a operação em processos de filtração de aerossóis, é interessante que haja uma dependência linear entre a queda de pressão $(\Delta \mathrm{P})$ e a velocidade superficial do gás $\left(\mathrm{v}_{\mathrm{s}}\right)$, de modo que o consumo energético seja minimizado, mesmo durante as oscilações de vazão do processo. Uma vez que a densidade e a viscosidade do fluido estão fixadas pela temperatura e pressão da corrente gasosa, então a redução em Fo pode ser conseguida pela redução na velocidade do escoamento ou pelo aumento na razão $k_{1} / k_{2}$. A primeira opção deve ser descartada, uma vez que implica no aumento da área de filtração, conforme previamente discutido. Desta forma, a opção é a otimização da razão $k_{1} / k_{2}$ através de alterações nas características estruturais da membrana filtrante. Felizmente, ou não, os dados disponíveis na literatura indicam que as constantes $\mathrm{k}_{1} \mathrm{e}$ $\mathrm{k}_{2}$ não são independentes entre si, mas estão correlacionadas por uma única e clara tendência, não importando o tipo de meio poroso (granular, celular ou fibroso) [2]. Embora não haja uma explicação física satisfatória para essa tendência até o momento, ela é útil quando valores experimentais de $\mathrm{k}_{2}$ não estão disponíveis. Assim:

$$
\mathrm{k}_{2}=\exp \left(-\frac{1,71588}{\mathrm{k}_{1}^{0,08093}}\right)
$$

A substituição da Equação (G) na Equação (A) fornece:

$$
\frac{\Delta \mathrm{P}}{\mathrm{L}}=\frac{\mu}{\mathrm{k}_{1}} \mathrm{v}_{\mathrm{s}}+\frac{\rho}{\exp \left(-\frac{1,71588}{\mathrm{k}_{1}^{0,08093}}\right)} \mathrm{v}_{\mathrm{s}}^{2}
$$

\section{PROCEDIMENTO EXPERIMENTAL}

\section{Avaliação dos requisitos de permeabilidade de um filtro cerâmico}

$\mathrm{O}$ conjunto de equações A-H pode ser usado para estabelecer os valores de $\mathrm{k}_{1}$ e $\mathrm{k}_{2}$ necessários para minimizar a queda de pressão para uma dada velocidade de filtração ou maximizar a velocidade para uma restrição na queda de pressão. O seguinte procedimento é proposto para membranas filtrantes planas (placas ou discos): i) Obtenção dos parâmetros operacionais da corrente gasosa durante a filtração: temperatura $T$, pressão $P$ e vazão volumétrica $Q$; ii) Cálculo das propriedades do gás ( $\mu$ e $\rho$ ) para as condições operacionais; iii) Definição de uma queda de pressão máxima para a membrana limpa (usualmente $\Delta \mathrm{P}<1000$ Pa); iv) Definição de uma velocidade superficial mínima para o processo (em geral, $\left.\left.\mathrm{v}_{\mathrm{s}}>0,01 \mathrm{~m} / \mathrm{s}\right) ; \mathrm{v}\right)$ Definição de uma espessura máxima para a membrana porosa (em geral, $\mathrm{L}<0,03 \mathrm{~m} / \mathrm{s}$ ); vi) Uso da equação $\mathrm{H}$ para determinar $\mathrm{o}$ valor requerido para a constante $\mathrm{k}_{1}$; vii) Uso da equação $\mathrm{G}$ para determinar o valor requerido para a constante $\mathrm{k}_{2}$; viii) Verificação dos pesos dos termos linear e parabólico na equação de Forchheimer através do parâmetro Fo; ix) Uso dos valores de $\mathrm{k}_{1}, \mathrm{k}_{2}$, L, $\mu$ e $\rho$ na equação A para simular outras condições de escoamento $\left(\Delta \mathrm{P} \mathrm{x} \mathrm{v}_{\mathrm{s}}\right)$ de interesse; $\left.\mathrm{x}\right)$ Cálculo da área total requerida de filtração $\mathrm{A}\left(\mathrm{A}=\mathrm{Q} / \mathrm{v}_{\mathrm{s}}\right)$; xi) Cálculo do número de membranas porosas requeridas, com base nas dimensões e área unitária de cada membrana $\mathrm{A}_{\mathrm{i}}\left(\mathrm{n}=\mathrm{A} / \mathrm{A}_{\mathrm{i}}\right)$. Como exemplo de tal procedimento, a Fig. 1a-b mostra o intervalo de valores de $\mathrm{k}_{1}$ e $\mathrm{k}_{2}$ necessários para elementos cerâmicos porosos de diferentes espessuras para resultar em queda de pressão de $1000 \mathrm{~Pa}$ durante o escoamento de ar a $25^{\circ} \mathrm{C}$ e 1 atm $(0,1 \mathrm{MPa})$. Observa-se na Fig. 1c que mesmo para $\mathrm{v}_{\mathrm{s}}=20 \mathrm{~cm} / \mathrm{s}$, a queda de pressão 
apresenta uma dependência razoavelmente linear com a velocidade (Fo $<<1)$. Portanto, embora ambas as constantes $\mathrm{k}_{1}$ e $\mathrm{k}_{2}$ estejam disponíveis para a análise, nesta condição de escoamento a lei de Darcy é válida e apenas o termo linear $\left[\mu v_{\mathrm{s}} / \mathrm{k}_{1}\right]$ é importante para caracterizar a permeação pela
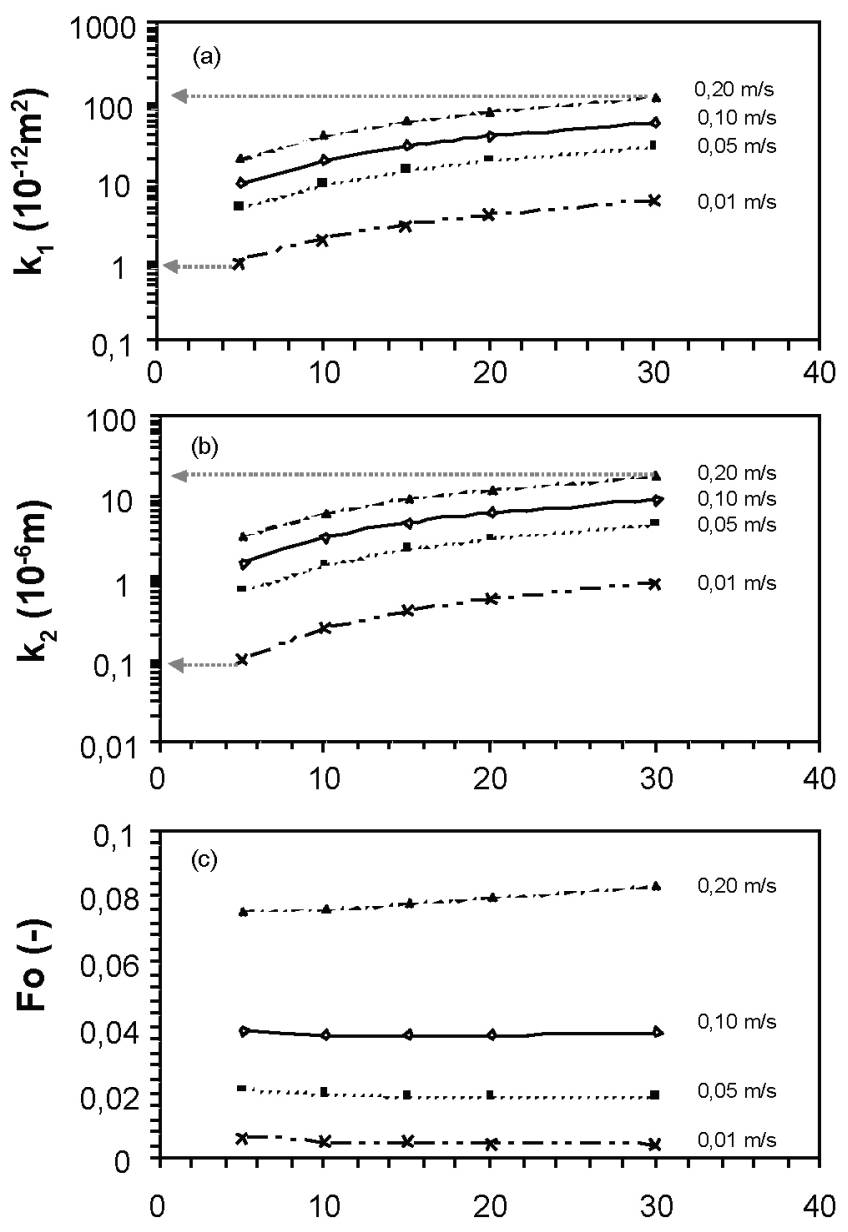

Espessura média $(\mathrm{mm})$

Figura 1: Faixas de valores de $\mathrm{k}_{1}$ e $\mathrm{k}_{2}$ requeridos para atingir uma queda de pressão de $1000 \mathrm{~Pa}$ (em membranas cerâmicas planas) em condições ambientes de escoamento de ar. (c) Valores de Fo.

[Figure 1: Required ranges of $k_{1}$ and $k_{2}$ for ceramic membranes $\Delta P$ $=1000 \mathrm{~Pa}$ and airflow at ambient conditions. (c) Fo values.]

membrana.

Obviamente que para condições de escoamento em altas temperaturas e pressões, as propriedades do gás, $\mu$ e $\rho$ devem ser corrigidas, alterando o intervalo requerido para a otimização de $\mathrm{k}_{1}$ e $\mathrm{k}_{2}$. Por exemplo, para escoamento de ar a $800{ }^{\circ} \mathrm{C}$ e 8 atm, espera-se um aumento por um fator de 2,5 na viscosidade e de 2,2 na densidade do ar, de modo que as curvas na Fig. 1 devem ser recalculadas para manter a queda de pressão desejada inalterada.

A Fig. 2 mostra um mapa que correlaciona os valores experimentais de $\mathrm{k}_{1}$ e $\mathrm{k}_{2}$ disponíveis na literatura. A região marcada localiza o intervalo de permeabilidade requerido para a produção de membranas cerâmicas para a filtração de aerossóis em temperatura ambiente e também a $800{ }^{\circ} \mathrm{C}$.

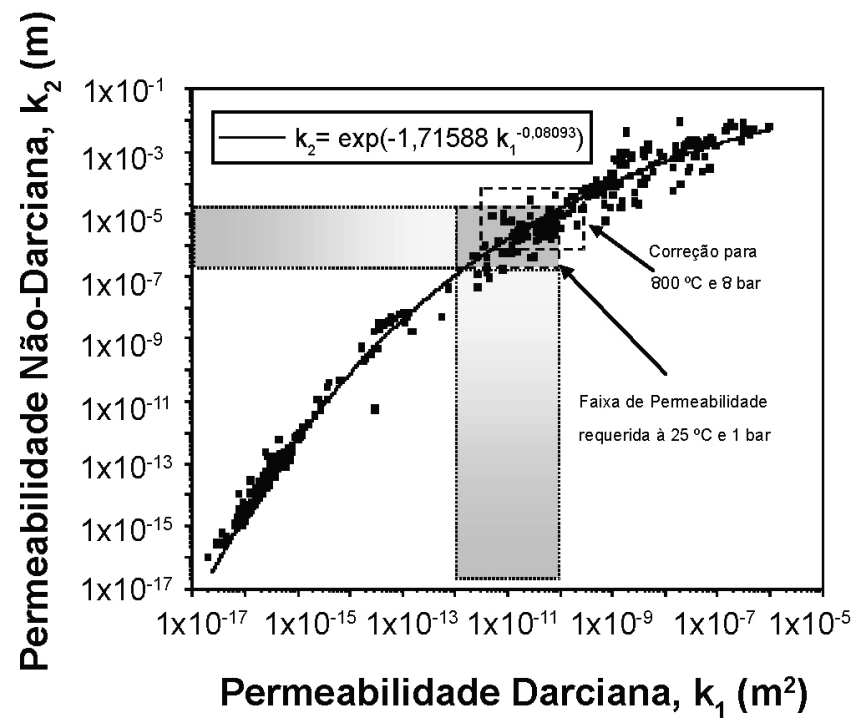

Figura 2: Faixa de permeabilidade requerida para membranas cerâmicas para filtração de aerossóis.

[Figure 2: Required permeability ranges of flat ceramic membranes for aerosol filtration.]

Conforme mencionado anteriormente, embora as constantes $\mathrm{k}_{1}$ e $\mathrm{k}_{2}$ não variem conforme o tipo de fluido ou com as condições de escoamento, elas podem depender da temperatura de operação, devido às alterações nos poros causadas por dilatação térmica da estrutura cerâmica. Em geral, a literatura cita um aumento de $\mathrm{k}_{1}$ com a temperatura, enquanto que $\mathrm{k}_{2}$ apresenta uma tendência relativamente errática, pelo menos na faixa típica de filtração [4]. Tipicamente, é esperado um aumento de permeabilidade com a temperatura, que pode ser ou não compensado pelo aumento da viscosidade e da densidade do ar nas condições operacionais [4, 11]. Para minimizar essa variação de comportamento, é interessante o uso de composições cerâmicas com baixo coeficiente de dilatação térmica. De todo modo, é recomendado que haja uma avaliação experimental dos valores de $\mathrm{k}_{1}$ e $\mathrm{k}_{2}$ nas condições reais de filtração para quantificar o efeito global das alterações estruturais e fluidodinâmicas sobre a queda de pressão.

\section{Geometria do elemento filtrante}

Os testes em laboratório para a confirmação experimental das constantes de permeabilidade $\mathrm{k}_{1}$ e $\mathrm{k}_{2}$ para uma dada composição cerâmica são usualmente realizadas com amostras planas, como discos ou placas (Fig. 3a), para as quais as equações A-H e o procedimento de otimização de permeabilidade anteriormente descrito é válido. Contudo, a prática industrial requer geometrias de elementos que maximizem a área exposta à filtração no menor volume possível, tais como nos casos de cartuchos e colméias [1- 
2, 13], bem como geometrias que facilitem a limpeza automática e manutenção periódica, tais como nos casos de velas e tubos (Fig. 3b) [1-3]. Neste último caso, a integração da equação de Forchheimer em coordenadas cilíndricas para escoamento puramente radial resulta em:

$$
\frac{\Delta \mathrm{P}}{\left(\mathrm{D}_{\mathrm{o}} / 2\right)}=\frac{\mu_{\mathrm{o}}}{\mathrm{k}_{1}} \operatorname{Ln}\left(\frac{\mathrm{D}_{0}}{\mathrm{D}_{\mathrm{i}}}\right) \mathrm{v}_{\mathrm{so}}+\frac{\rho_{\mathrm{o}}}{\mathrm{k}_{2}}\left(\frac{\mathrm{D}_{0}-\mathrm{D}_{\mathrm{i}}}{\mathrm{D}_{\mathrm{i}}}\right) \mathrm{v}_{\text {so }}{ }^{2}
$$

na qual $\mathrm{D}_{\mathrm{i}}$ e $\mathrm{D}_{\mathrm{o}}$ são respectivamente os diâmetros interno e externo da vela cerâmica, $\mathrm{v}_{\mathrm{so}}$ é a velocidade baseada na área externa da vela $\left(\mathrm{v}_{\mathrm{so}}=\mathrm{Q} / \pi \mathrm{D} \mathrm{L} \mathrm{L}\right)$ e $\mu_{\mathrm{o}}$ e $\rho_{\mathrm{o}}$ são respectivamente a viscosidade e a densidade do gás nas condições de temperatura e pressão na face externa da vela cerâmica.

Desta forma, a equação I pode substituir as equações A ou F para o procedimento de otimização de permeabilidade previamente descrito, bem como a espessura L da membrana plana é substituída pelos diâmetros $\mathrm{D}_{\mathrm{i}}$ e $\mathrm{D}_{\mathrm{o}}$.

\section{Relação entre permeabilidade e características estruturais do elemento filtrante}

Uma vez estabelecidos os requisitos de permeabilidade para o processo de filtração, o próximo passo é o processamento da estrutura porosa cerâmica com os valores de $\mathrm{L}$ (ou $\mathrm{D}_{\mathrm{i}}$ e $\mathrm{D}_{\mathrm{e}}$ ) e de $\mathrm{k}_{1}$ e $\mathrm{k}_{2}$ desejados. Portanto, é necessário primeiramente avaliar como esses dois últimos parâmetros são qualitativamente afetados por outras características mensuráveis da estrutura porosa:

- Porosidade $(\varepsilon)$ : o aumento na fração volumétrica de poros reduz a velocidade intersticial e aumenta as constantes de permeabilidade. Contudo, apenas a porosidade aberta e interconectada contribui para o escoamento, o que não é fácil de ser avaliado [14]. Modelos simplificados assumem (a)
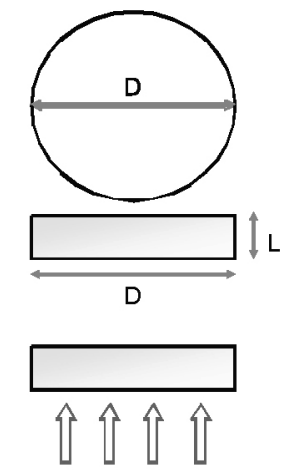

Direção do fluxo (b)

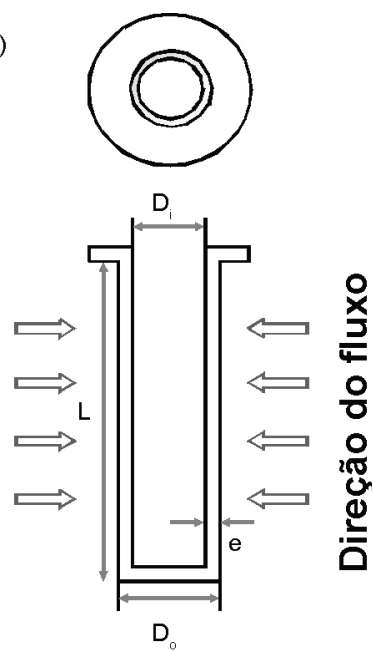

Figura 3: Geometrias típicas dos elementos filtrantes. (a) disco e (b) vela.

[Figure 3: Typical geometries for filter elements. (a) disk; (b) candle.] uma influência linear de $\varepsilon$ sobre $\mathrm{k}_{1}$ e uma dependência quadrática sobre $\mathrm{k}_{2}$ para meios celulares [2, 14-15]. Para meios granulares não-consolidados, a dependência é mais complexa $\left(\left[\varepsilon^{3} /(1-\varepsilon)^{2}\right]\right.$ para $\mathrm{k}_{1}$ e $\left[\varepsilon^{3} /(1-\varepsilon)\right]$ para $\left.\mathrm{k}_{2}\right)$, porém bem comprovada experimentalmente [15]. A porosidade típica de filtros comerciais para aerossóis em altas temperaturas é em torno de 50 a $85 \%$, mas pode atingir $96 \%$ para elementos fibrosos [2-3, 13].

- Diâmetro de poros $\left(\mathrm{d}_{\text {poro }}\right)$ : O tamanho das partículas que podem ser coletadas pelo meio filtrante é determinado pelo tamanho do poro nas regiões de maior constrição. O modo ideal de filtração ocorre com a deposição das partículas sobre a superfície do elemento filtrante, após a formação de uma camada de pó, conhecida como torta de filtração $[4,13]$. O aumento do tamanho do poro facilita a penetração, deposição e acúmulo de pó na matriz porosa, com o progressivo entupimento do filtro ou então a redução da eficiência de coleta (quando as partículas de pó simplesmente atravessam toda a espessura do filtro e são eliminadas junto à corrente gasosa). Em relação ao escoamento do gás, o aumento do diâmetro de poro reduz a área de contato sólido-fluido, bem como diminui a tortuosidade do meio poroso, ambos fatores que aumentam as constantes de permeabilidade. Alguns modelos assumem que $\mathrm{k}_{1}$ e $\mathrm{k}_{2}$ variam respectivamente com $\mathrm{d}_{\text {poro }}{ }^{2}$ e $\mathrm{d}_{\text {poro }}$, porém essa tendência é controversa para meios cerâmicos celulares [2]. Tipicamente, o tamanho de poros em filtros cerâmicos comerciais varia de 0,1 a 10 $\mu \mathrm{m}$, podendo atingir $60 \mu \mathrm{m}$ para alguns meios fibrosos. Um aspecto interessante de otimização é que a matriz porosa pode ser processada com gradientes de distribuição de poros. Assim, na superfície em contato com o aerossol, uma camada de pequena espessura com poros pequenos facilita a retenção superficial de pó, mas apresenta baixa resistência ao escoamento (baixos $\mathrm{k}_{1}, \mathrm{k}_{2} \mathrm{e} \mathrm{L}$ ). Essa camada, por apresentar pequena espessura, tende a ser frágil e pode ser suportada por uma camada de maior espessura, que por não ter a finalidade de reter as partículas pode apresentar maiores valores de $\mathrm{k}_{1}$ e $\mathrm{k}_{2}$, de modo a também apresentar baixa queda de pressão. Essa estrutura em dupla camada (ou em multi-camadas) tem sido uma das opções discutidas na literatura para a otimização de filtros cerâmicos celulares para aerossóis $[4,16]$.

O projetista ou o produtor dos elementos filtrantes tem então duas opções para otimizar a permeabilidade da estrutura cerâmica baseadas nas condições de processamento: i) Previsão das constantes de permeabilidade $k_{1}$ e $k_{2}$ baseada em outras propriedades estruturais do meio poroso que estão intimamente relacionadas às condições de processamento, como a porosidade e o tamanho de poros; ii) Avaliação experimental de $\mathrm{k}_{1}$ e $\mathrm{k}_{2}$ em amostras e associação direta com as variáveis de processamento cerâmico, incluindo a composição cerâmica, características da suspensão, tratamento térmico, etc.

Infelizmente, não há na literatura técnica até o momento concordância sobre relações matemáticas confiáveis e genéricas que permitam a previsão de $\mathrm{k}_{1}$ e $\mathrm{k}_{2}$ baseadas na porosidade permeável e no tamanho de poros. Além disso, 
Tabela I - Composições das cerâmicas porosas testadas neste trabalho.

[Table I - Compositions of porous bodies tested in this work.]

\begin{tabular}{|c|c|c|c|c|c|c|c|c|c|c|c|c|c|c|c|}
\hline \multirow{3}{*}{ Matéria-prima } & \multicolumn{15}{|c|}{ Amostra } \\
\hline & \multicolumn{15}{|c|}{ Suspensão } \\
\hline & 1 & 2 & 3 & 4 & 5 & 6 & 7 & 8 & 9 & 10 & 12 & 13 & 14 & $1 \mathrm{~S}^{8}$ & $2 \mathrm{~S}^{8}$ \\
\hline A17 NE (\%) & & & & & & & & 10,0 & & & & & & & \\
\hline CT 3000SG (\%) & & & & & & & & 65,0 & & & & & & & \\
\hline Hidrogard $(\%)^{1}$ & & & & & & & & 25,0 & & & & & & & \\
\hline Água $(\%)^{2}$ & & & & & & & & $=82$, & & & & & & & \\
\hline Poliacrilato de sódio $(\%)^{3}$ & & & & & & & & 1,0 & & & & & & & \\
\hline $\operatorname{EVA}(\%)^{3,4}$ & 0,0 & 0,0 & 0,0 & 0,0 & 4,0 & 4,0 & 4,0 & 4,0 & 4,0 & 4,0 & 4,0 & 4,0 & 4,0 & 4,0 & 4,0 \\
\hline Fibra de PP $(\%)^{3}$ & 0,0 & 0,0 & 0,0 & 0,0 & 0,0 & 0,0 & 0,0 & 0,0 & 0,075 & 0,150 & 0,0 & 0,0 & 0,0 & 0,0 & 0,0 \\
\hline Fibra de celulose $(\%)^{3}$ & 0,0 & 0,0 & 0,0 & 0,0 & 0,0 & 0,0 & 0,0 & 0,0 & 0,0 & 0,0 & 0,0 & 0,05 & 0,05 & 0,05 & 0,05 \\
\hline \multirow[t]{2}{*}{$\mathrm{PVA}^{3,5}$} & 0,0 & 0,0 & 0,0 & 0,0 & 0,0 & 0,0 & 0,0 & 0,0 & 0,0 & 0,0 & 0,0 & 0,0 & 0,05 & 0,0 & 0,05 \\
\hline & \multicolumn{15}{|c|}{ Espuma } \\
\hline Água $(\%)^{3}$ & \multicolumn{15}{|c|}{47,6} \\
\hline $\operatorname{LESS}(\%)^{3,6}$ & \multicolumn{15}{|c|}{4,8} \\
\hline Glicerina $(\%)^{3}$ & \multicolumn{15}{|c|}{31,7} \\
\hline Poliacrilato de sódio $(\%)^{3}$ & \multicolumn{15}{|c|}{15,9} \\
\hline $\operatorname{EVA}(\%)^{3}$ & 0,0 & 0,0 & 0,0 & 0,0 & 0,0 & 0,0 & 0,0 & 0,0 & 0,0 & 0,0 & 2,0 & 2,0 & 2,0 & 0,0 & 0,0 \\
\hline & \multicolumn{15}{|c|}{ Consolidador } \\
\hline $\mathrm{CA} 14^{7}$ & \multicolumn{15}{|c|}{1,5} \\
\hline $\mathrm{V}_{\text {espuma }} / \mathrm{V}_{\text {suspensão }}$ & 0,0 & 0,5 & 1,0 & 1,5 & 0,0 & 0,5 & 1,0 & 1,5 & 1,0 & 1,0 & 1,0 & 1,0 & 1,0 & 1,0 & 1,0 \\
\hline
\end{tabular}

${ }^{1}$ Hidróxido de alumínio, Alcoa, USA; ${ }^{2}$ Porcentagem baseada na massa de sólidos $\phi=m s /(m s+m l) ;{ }^{3}$ Porcentagem baseada na massa sólidos A17 e CT $3000 S G$ e Hidrogard; ${ }^{4}$ Etileno vinil acetato; ${ }^{5}$ Poli vinil acetato; ${ }^{6}$ Lauril éter sulfato de sódio; ${ }^{7}$ Cimento de aluminado de cálcio, Alcoa, USA; ${ }^{8}$ Amostras cortadas.

a própria avaliação desses dois parâmetros é muito mais complicada do que a avaliação experimental das constantes de permeabilidade [2]. Por essa razão, neste trabalho, nenhuma tentativa de correlacionar a permeabilidade e outras propriedades estruturais foi realizada e as constantes $\mathrm{k}_{1}$ e $\mathrm{k}_{2}$ foram obtidas diretamente dos experimentos com escoamento de ar.

Amostras de cerâmica porosa foram preparadas [5], na qual uma espuma aquosa estável foi adicionada em uma suspensão cerâmica à base de alumina. As matérias-primas e as proporções utilizadas nas suspensões e nas espumas são mostradas na Tabela I.

Para preparação da suspensão cerâmica o pó foi adicionado no líquido e misturado em $500 \mathrm{rpm}$ em um misturador IKA, Labortechnik RW 20 e, em seguida, os aglomerados foram rompidos em um dispersor de alta energia de cisalhamento (IKA, Labortechnik-T25 Basic) em 9500 rpm.

A espuma aquosa estável foi preparada misturando os materiais descritos na Tabela I sob alta rotação em um copo de becker até adquirir a consistência desejada. A suspensão e a espuma foram misturadas em diferentes proporções e homogeneizadas com cuidado para evitar mudanças no tamanho das bolhas. Finalmente, foi adicionado o agente consolidador sob rotação de $800 \mathrm{rpm}$ (IKA, Labortechnik=RW 20) e a suspensão foi misturada por mais alguns segundos, sendo moldados, em seguida, os corpos-de-prova. As amostras foram moldadas em discos (Fig. 3a) com diâmetro de $5 \mathrm{~cm}$ e espessura de 0,7 a $1,0 \mathrm{~cm}$ e em cilindros com diâmetros de $1,8 \mathrm{~cm}$ e altura de $2,7 \mathrm{~cm}$. Após a secagem por $24 \mathrm{~h}$ em temperatura ambiente, as amostras foram queimadas em $1200{ }^{\circ} \mathrm{C}$ for $2 \mathrm{~h}$, com taxa de aquecimento $5{ }^{\circ} \mathrm{C} / \mathrm{min}$.

Adistribuição dos poros foi caracterizada por porosimetria de intrusão de mercúrio (Quantachrome Instruments) e a porosidade foi quantificada a partir do ensaio de Arquimedes (NBR 9778). A resistência mecânica foi determinada por tração na compressão diametral seguindo a norma técnica NBR 7222/94 em uma máquina universal de ensaios (Instron 5569). A avaliação da microestrutura foi realizada por microscopia eletrônica de varredura em um microscópio LEO (Stereoscan 440) acoplado a um espectrômetro de dispersão de energia (EDS), com as amostras recobertas por platina.

A permeabilidade foi avaliada nas condições ambientes ( $\mathrm{T} \sim$ $\left.25^{\circ} \mathrm{C}, \mathrm{P}_{\text {atm }} \sim 715 \mathrm{mmHg}, \mu \sim 1,86 \times 10^{-5} \mathrm{~Pa} . \mathrm{s}, \rho \sim 1,11 \mathrm{~kg} / \mathrm{cm}^{3}\right)$ em 3 corpos-de-prova de cada composição (área de escoamento $5,15 \mathrm{~cm}^{2}$ ). Durante os experimentos foram avaliadas as vazões (Q) de ar obtidas em resposta às variações das pressões de entrada de ar $\left(\mathrm{P}_{\mathrm{e}}\right)$ aplicadas. A vazão $\mathrm{Q}$ foi quantificada a partir do deslocamento de bolhas de sabão no interior de uma pipeta 


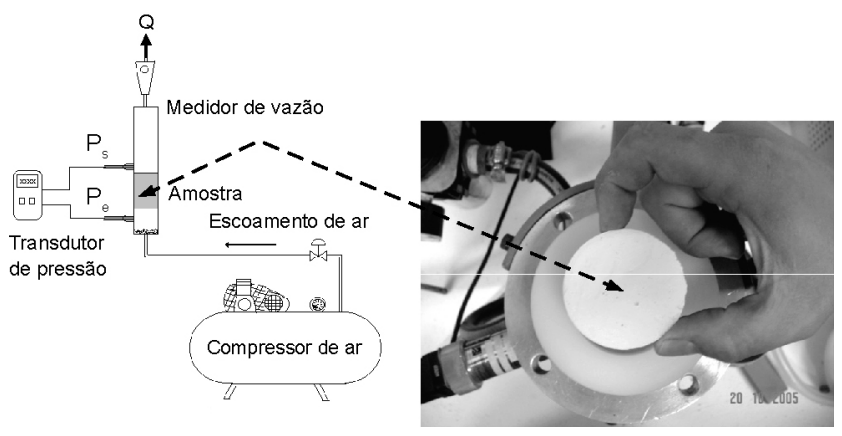

Figura 4: Detalhes do aparato para avaliação experimental de permeabilidade das amostras cerâmicas.

[Figure 4: Details of permeability apparatus for ceramics.]

graduada (0-15 L/min) e/ou com a utilização de rotâmetros (0-100 L/min) e a queda de pressão através da amostra foi acompanhada com transdutores eletrônicos de pressão (0-10 bar). As constantes de permeabilidade $\mathrm{k}_{1}$ e $\mathrm{k}_{2}$ foram obtidas por um ajuste polinomial dos dados experimentais através da equação de Forchheimer (equação A) de acordo com o método dos mínimos quadrados. A equação $C$ foi utilizada para obter a queda de pressão $\Delta \mathrm{P}$, devido à grande faixa de $\mathrm{P}_{\mathrm{e}}$ utilizada nos testes. A Fig. 4 ilustra o equipamento utilizado para a avaliação experimental da permeabilidade das amostras cerâmicas neste trabalho.

\section{RESULTADOS E DISCUSSÃO}

Cerâmicas porosas podem ser processadas por diversas técnicas, sendo que, dentre elas podem ser citados o método da réplica da esponja polimérica, gelcasting de espumas cerâmicas, queima de partículas orgânicas, e mais recentemente desenvolvida, a técnica da adição de espumas aquosas em suspensões cerâmicas. Esta última tem mostrado grandes vantagens com relação às demais, devido ao fato de permitir a produção de corpos cerâmicos com elevados níveis de porosidade e distribuição estreita de poros, tornando a microestrutura das cerâmicas homogênea, conforme apresentado nas microscopias ilustradas na Fig. 5.

A Fig. 5a ilustra a microestrutura da amostra 1 (sem adição de espuma aquosa), com a composição ilustrada na Tabela I, enquanto que as Figs. $5 b$ e 5 c apresentam as microscopias das amostras 3 e $2 \mathrm{~S}$, respectivamente, ilustrando os efeitos da incorporação da espuma aquosa na suspensão cerâmica. As diferenças causadas na distribuição de poros pela adição de espuma aquosa ou pela variação da relação entre o volume de espuma e o volume de suspensão são ilustradas na Fig. 6.

$\mathrm{Na}$ amostra 1 o trajeto para a percolação do ar é devido somente aos pequenos vazios interparticulares, inicialmente preenchidos pela água utilizada no processamento da cerâmica e posteriormente pelos produtos de hidratação do cimento. Quando as bolhas são incorporadas na matriz a partir da adição da espuma aquosa, é gerada uma população de poros na estrutura cerâmica, com diâmetros variando entre $10 \mu \mathrm{m}$ e $100 \mu \mathrm{m}$. Já com o aumento do tamanho e da
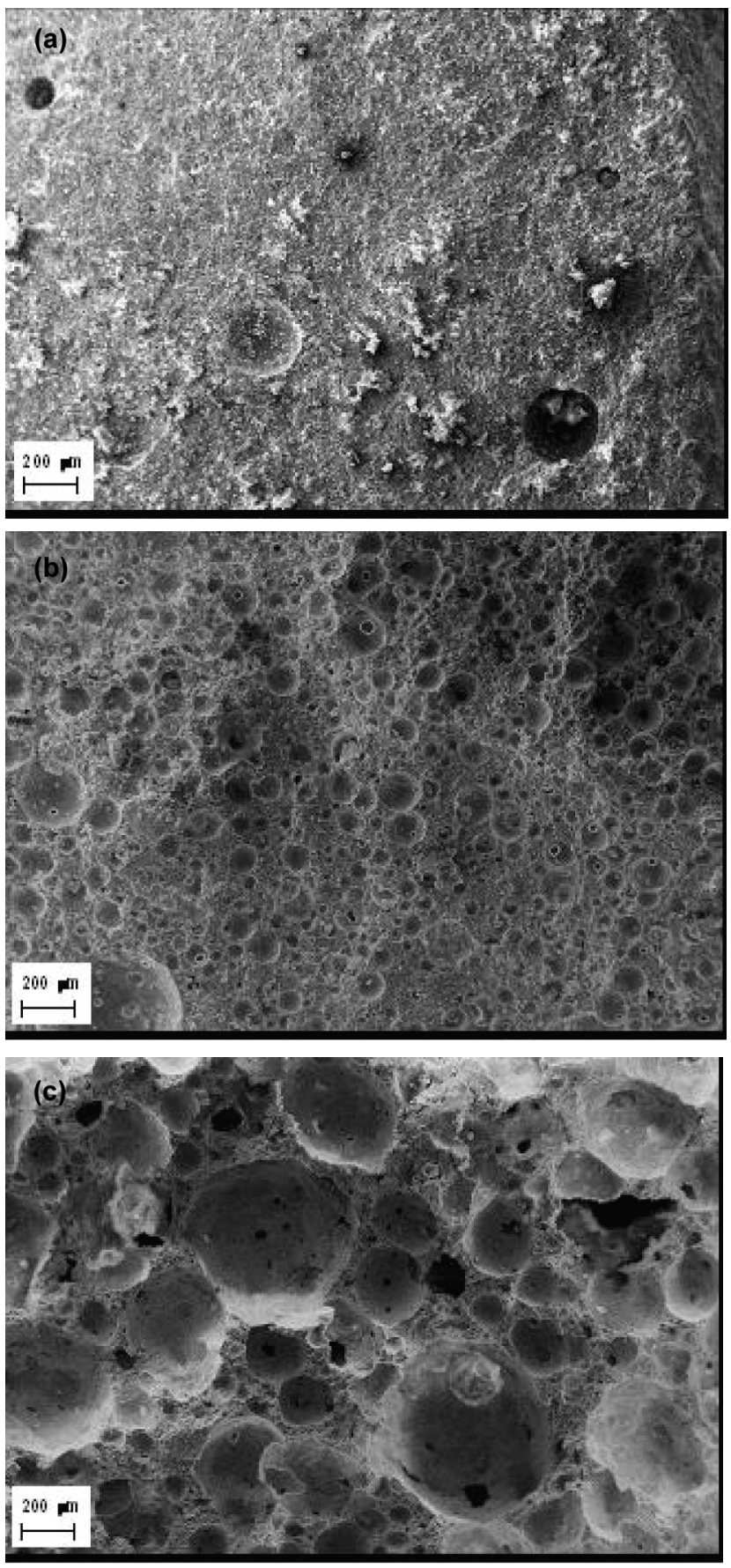

Figura 5: Micrografias obtidas por microscopia eletrônica de varredura das amostras: (a) Amostra 1; (b) 3 e (c) $2 \mathrm{~S}$.

[Figure 5: SEM micrographs of samples: (a) batch 1; (b) batch 3; (c) batch $2 S$.

quantidade de bolhas, há a criação de interconexão entre as células (janelas), que resultam em distribuições bimodais de poros, conforme pode ser notado nas curvas referentes às amostras 13 e $2 \mathrm{~S}$. Entretanto, as mudanças na distribuição de tamanhos de poros não foram atingidas somente com o aumento do volume de espuma aquosa na suspensão, mas também devido à presença de outros aditivos, conforme apresentado na Tabela I.

Os efeitos da incorporação de espuma no tamanho médio 


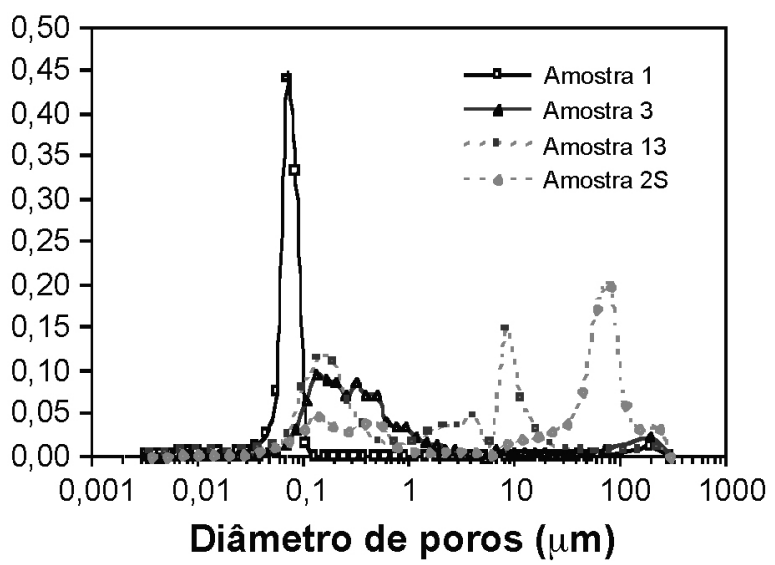

Figura 6: Distribuição de poros de algumas amostras processadas neste trabalho.

[Figure 6: Typical pore size distributions for some of the tested batches.]

de poros de todas as amostras avaliadas no trabalho são mais bem descritos na Fig. 7.

A proximidade entre os valores da média e da moda dos poros é uma conseqüência da estreita distribuição de poros produzidas nas amostras, o que consiste em uma das vantagens desta técnica de processamento. Vale a pena lembrar que mesmo não tendo sido adicionada espuma na amostra 5, a presença de aditivo (EVA) foi responsável por um pequeno aumento na porosidade, já que este material, além de gerar poros após a queima (devido à sua decomposição) pode gerar bolhas de ar durante o processamento da suspensão. Isso explica as maiores porosidades, quando comparadas com a amostra 1 . De acordo com o esperado na análise de porosimetria de mercúrio e observado nas Figs. 5-7, a distribuição de poros esteve mais relacionada com o tamanho das interconexões (janelas) entre os poros do que com o diâmetro das células.

A fração de poros abertos e fechados em cada amostra pode ser observada na Fig. 8.

A densidade real dos materiais sólidos secos foi

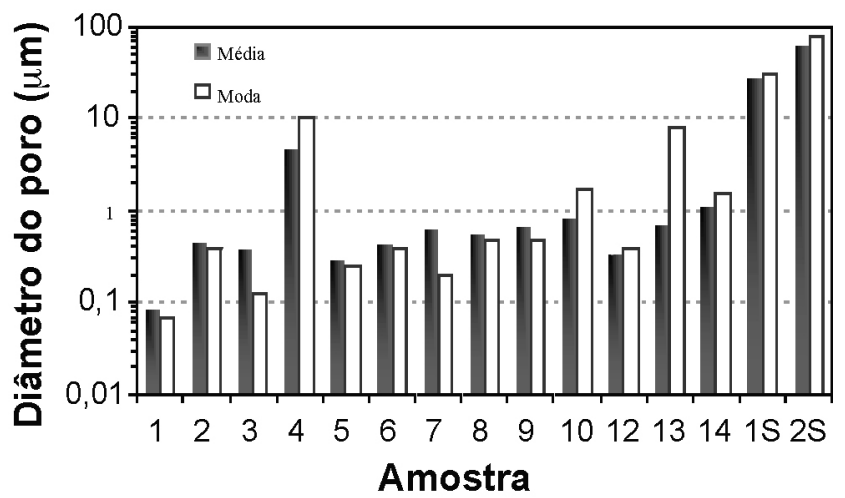

Figura 7: Diâmetros médios de poros das amostras testadas. [Figure 7: Average pore sizes of tested batches.]

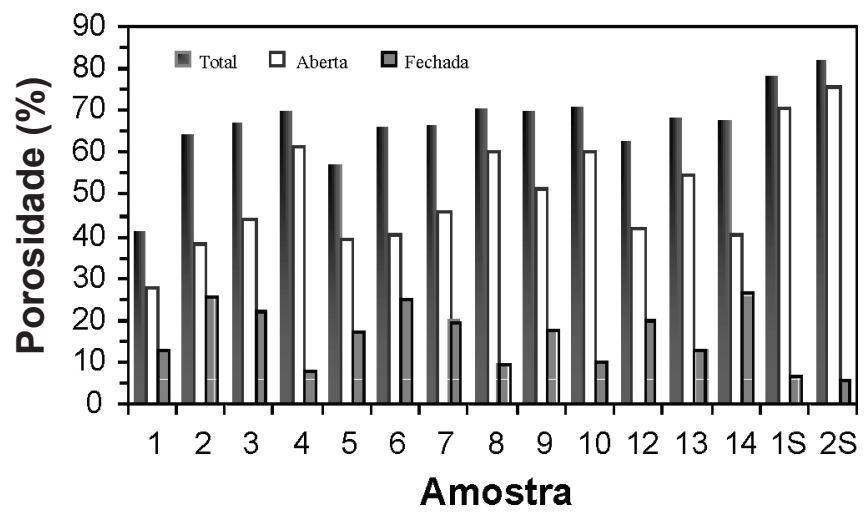

Figura 8: Níveis de porosidade das amostras testadas.

[Figure 8: Porosity levels of tested batches.]

determinada por picnometria de gás hélio o valor encontrado foi de $3,8 \mathrm{~g} / \mathrm{cm}^{3}$. A adição de espuma aquosa na suspensão cerâmica foi responsável pelo aumento da porosidade total dos materiais, variando de cerca de $62 \%$ para $82 \%$, atingido na amostra $2 \mathrm{~S}$. No entanto, mesmo nas amostras com altos níveis de porosidade total, a fração de poros fechados foi elevada ( 25\%), por exemplo, nas amostras 6 e 14. Isso indica que a presença de células isoladas (sem janelas) no interior da matriz não contribui para o escoamento do fluido e somente reduzem a resistência mecânica das cerâmicas. Como a proposta deste trabalho é de gerar cerâmicas com elevadas porosidades, mas também com boa conectividade entre os poros, certamente a utilização de fibras e de aditivos orgânicos aliada à incorporação de espuma aquosa deve funcionar bem para produzir este efeito.

A resistência mecânica $\left(\sigma_{\mathrm{f}}\right)$ das amostras é apresentada na Fig. 9.

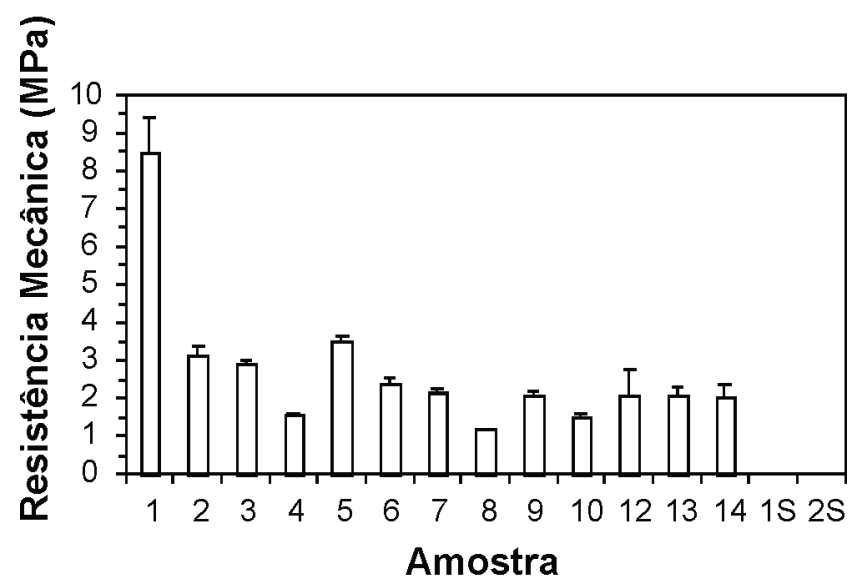

Figura 9: Resistência mecânica das amostras testadas. [Figure 9: Fracture strength of tested batches.]

Conformeesperado, os maiores valores foram encontrados nas amostras sem adição de espumas aquosas (amostras $1 \mathrm{e}$ 5). Para as outras amostras, nas quais foi adicionada a espuma aquosa, $\sigma_{\mathrm{f}}$ variou de 1,8 a 3,2 $\mathrm{MPa}$. Não são apresentados 


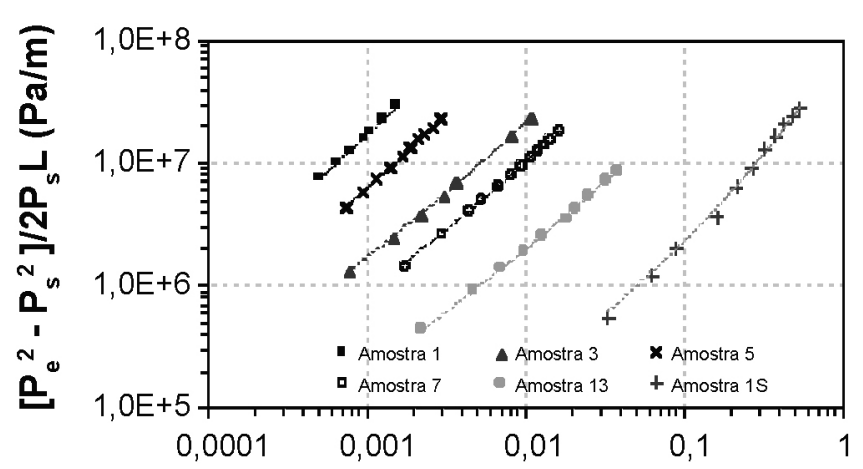

Velocidade superficial do ar, $v_{s}(\mathrm{~m} / \mathrm{s})$

Figura 10: Curvas típicas obtidas nos testes de permeabilidade. As linhas referem-se ao ajuste polinomial da equação de Forchheimer.

[Figure 10: Typical curves obtained in permeability tests. Lines refer to polynomial fitting of Forchheimer's equation.]

resultados de resistência mecânica para as amostras $1 \mathrm{~S}$ e $2 \mathrm{~S}$ porque essas amostras foram cortadas antes da realização dos testes. Os valores citados em literatura para a resistência mecânica de filtros cerâmicos comerciais (velas) são de 0,4 a 1,8 $\mathrm{MPa}[3,13,16]$, e de placas cerâmicas sinterizadas em $1100{ }^{\circ} \mathrm{C}$ em torno de 3,0 $\mathrm{MPa}[1,13]$. No presente trabalho, uma melhoria na resistência pode ser alcançada a partir do aumento do tempo de queima ou da temperatura de sinterização, mas isto pode provavelmente sacrificar a permeabilidade dos filtros.

Algumas das curvas experimentais de queda de pressão para a avaliação das constantes de permeabilidade $\mathrm{k}_{1}$ e $\mathrm{k}_{2}$ são apresentadas na Fig. 10. A utilização de escala logarítmica nos dois eixos reflete a grande variação nos resultados de permeabilidade.

A grande variação da permeabilidade pode ser confirmada na Fig. 11, onde os valores médios de $\mathrm{k}_{1}$ e $\mathrm{k}_{2}$ de todas as amostras são apresentados.

As menores permeabilidades foram observadas nas amostras 1 e 5, nas quais não foi adicionada espuma aquosa, enquanto que as amostras $1 \mathrm{~S}$ e $2 \mathrm{~S}$ apresentaram as maiores permeabilidades. Variações de 3 ordens de magnitude foram observadas na constante de permeabilidade Darciana $\left(\mathrm{k}_{1}\right)$, enquanto que variações de 5 ordens de magnitude foram observadas para a constante de permeabilidade não-Darciana $\left(\mathrm{k}_{2}\right)$, somente devido às manipulações das composições e proporções de espuma aquosa nas suspensões. Conforme esperado, houve uma correspondência clara de $\mathrm{k}_{1}$ e $\mathrm{k}_{2}$ com $\mathrm{d}_{\text {poro }}$ e com $\varepsilon$ e inversa com a resistência mecânica.

Um aspecto importante da otimização de membranas para a filtração é que a carga máxima admitida sobre a membrana não tem uma relação linear com a sua espessura; conseqüentemente, aumentando-se a espessura da membrana, a solicitação mecânica é reduzida rapidamente. Por outro lado, a queda de pressão aumenta linearmente com a espessura das membranas. Como os aumentos de $\mathrm{k}_{1}$ são usualmente relacionados com um decréscimo de $\sigma_{\mathrm{f}}$, então

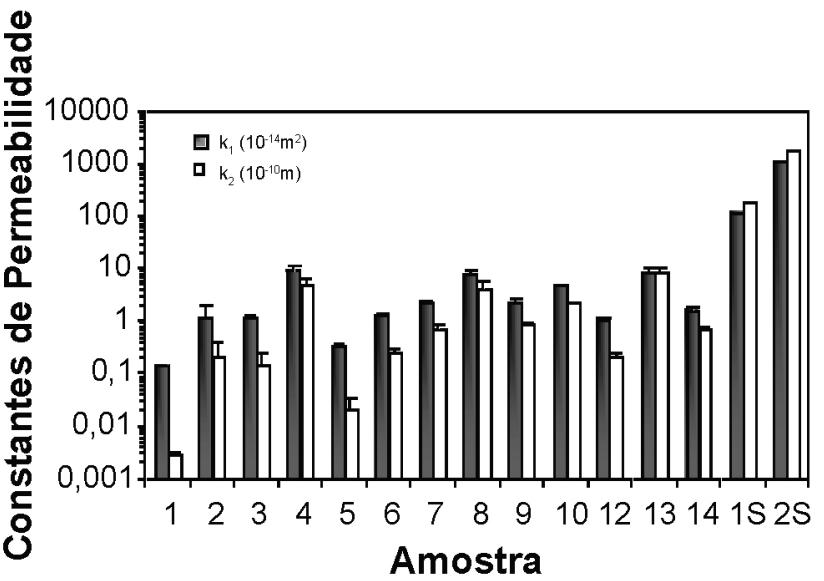

Figura 11: Parâmetros de permeabilidade $\mathrm{k}_{1}$ e $\mathrm{k}_{2}$ das amostras testadas.

[Figure 11: Permeability parameters $k_{1}$ and $k_{2}$ of tested batches.]

é possível, mas não é fácil, otimizar uma espessura média que seja adequada para minimizar tanto a queda de pressão quanto a tensão imposta sobre a estrutura.

A Fig. 12 apresenta uma série de resultados $\mathrm{k}_{1}$ e $\mathrm{k}_{2}$ das membranas cerâmicas produzidas neste trabalho, comparando-se com resultados de $\mathrm{k}_{1}$ e $\mathrm{k}_{2}$ de filtros para gases, obtidos em literatura.

De acordo com as Figs. 11 e 12 somente a amostra $2 \mathrm{~S}$ apresentou requisitos para ser utilizada em filtração de gases, seguida das amostras $8,1 \mathrm{~S}$ e 13 , conforme a especificação desejada nas Figs. 1 e 2.

Dados experimentais de $\mathrm{k}_{1}$ e $\mathrm{k}_{2}$ da amostra $2 \mathrm{~S}$ e a equação I foram utilizados para simular as curvas de queda de pressão

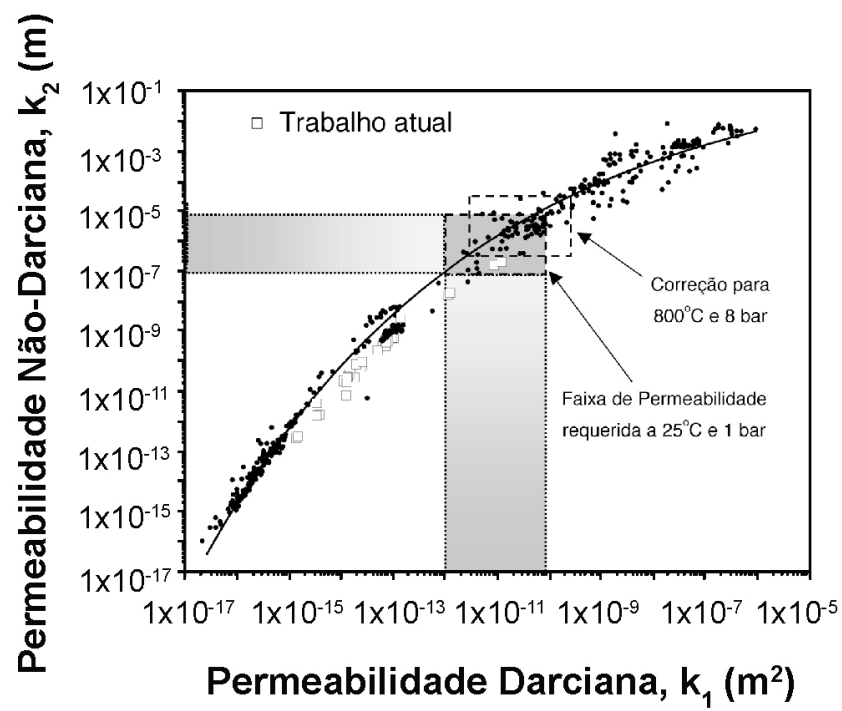

Figura 12: Comparação das constantes de permeabilidade obtidas neste trabalho, com as permeabilidades requeridas para filtros de aerossóis.

[Figure 12: Comparison of experimental permeability constants of produced membranes and the range required for aerosol filtration.] 

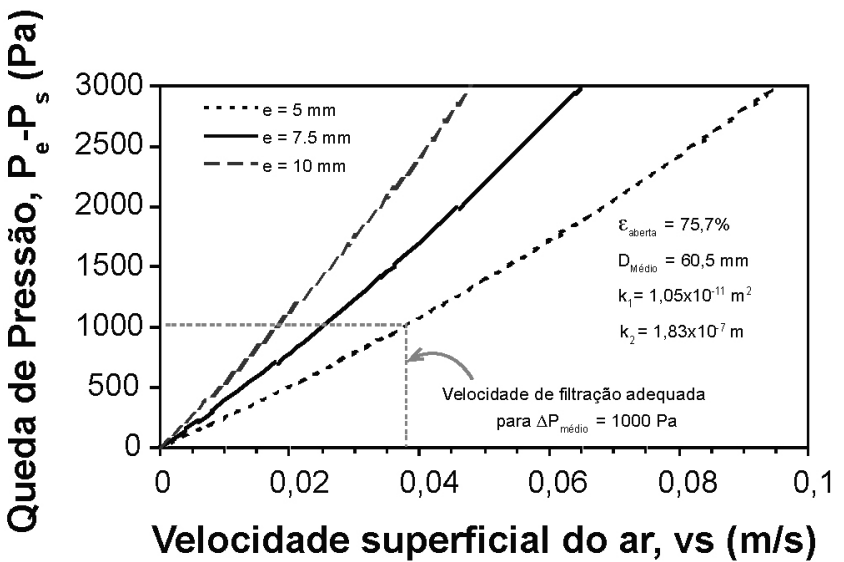

Figura 13: Simulação das curvas de queda de pressão para filtros de vela com diferentes espessuras de parede, com o diâmetro externo fixo $(60 \mathrm{~mm}), \mathrm{T}=800^{\circ} \mathrm{C}, \mathrm{P}=8$ bar.

[Figure 13: Simulated pressure drop curves for candles with different wall thickness e and fixed outside diameter $D_{o}=60 \mathrm{~mm}$. ( $\left.\left.T=800^{\circ} \mathrm{C}, \mathrm{P}=8 \mathrm{bar}\right).\right]$

resultantes em filtros do tipo vela hipotéticos e os resultados são apresentados na Fig. 13.

O diâmetro externo foi fixado em $60 \mathrm{~mm}$ e a espessura da parede foi variada de 5 a $10 \mathrm{~mm}$. As condições de escoamento de ar foram fixadas em $800{ }^{\circ} \mathrm{C}$ e 8 bar. Nesta situação, a velocidade de filtração deveria ser menor que $4 \mathrm{~cm} / \mathrm{s}$ para atender o limite de queda de pressão de $1000 \mathrm{~Pa}$ para o meio filtrante virgem. A compressibilidade do gás pode ser desprezada nesta situação $\left(\Delta \mathrm{P}=\mathrm{P}_{\mathrm{e}}-\mathrm{P}_{\mathrm{s}}\right)$, mas o mesmo não pode ser feito com os efeitos inerciais que correspondem a aproximadamente $10 \%$ do total da queda de pressão.

Embora a permeabilidade da amostra $2 \mathrm{~S}$ tenha atingido os requisitos para ser utilizada em filtros de gases em altas temperaturas, outros testes devem ser realizados para se avaliar se a expansão térmica em temperaturas acima de $800{ }^{\circ} \mathrm{C}$ irá influenciar nos valores das constantes de permeabilidade $\mathrm{k}_{1}$ e $\mathrm{k}_{2}$. De qualquer forma, a influência do diâmetro médio dos poros nesta composição $(\sim 60 \mu \mathrm{m})$ provavelmente poderia afetar negativamente a eficiência de coleta das partículas ou a limpeza dos elementos filtrantes em filtros de vela reais. De acordo com o método de processamento utilizado neste trabalho, estas características poderão ser otimizadas, adicionando-se uma grande quantidade de pequenas bolhas ou aumentando a quantidade de interconexões entre elas. A segunda opção parece mais promissora, já que podem ser adicionadas fibras orgânicas nas composições, sem causar grandes impactos na resistência mecânica dos filtros. As composições das amostras $8,13,1 \mathrm{~S}$ e $2 \mathrm{~S}$ parecem ser as mais fortes candidatas para desenvolvimentos de trabalhos futuros.

\section{CONCLUSÕES}

A otimização da permeabilidade é uma etapa crítica no desenvolvimento de filtros cerâmicos eficientes para o tratamento de aerossóis em temperaturas elevadas. Uma vez preenchidos os requisitos de resistência mecânica e eficiência de coleta, é a otimização da permeabilidade que garante uma redução da área total de filtração e minimiza o número dos elementos filtrantes necessários para o processo. Nesse contexto, o objetivo deste trabalho foi apresentar um critério claro que permita a otimização da permeabilidade de um filtro cerâmico baseado nas restrições operacionais de filtração (velocidade do ar e queda de pressão).

A técnica de incorporação de espumas em suspensões cerâmicas foi utilizada, pois permite a manufatura de corpos com elevada porosidade e distribuição estreita de tamanho de poros pequenos, duas das características essenciais para um filtro permeável, porém com boa resistência mecânica e eficiência de coleta. Diferentes composições foram testadas e os valores experimentais das variáveis de interesse foram confrontados com os valores desejados para uma boa operação. As composições mais permeáveis e com boa resistência mecânica foram selecionadas, de modo que possam passar por novo processo de otimização, cujo foco será a eficiência de coleta.

\section{AGRADECIMENTOS}

Ao MCT/CNPq, Procs. CT-Energ 401110/2004-4 e $551601 / 2005-1$, pelo financiamento deste trabalho.

\section{REFERÊNCIAS}

[1] D. Fino, G. Saracco, Gas (Particulate) Filtration. Cellular Ceramics: Structure, Manufacturing, Properties and Applications, Wiley-VCH, Weinheim - Germany (2005) 416.

[2] M. D. M. Innocentini, P. Sepulveda, F. Ortega, Permeability. Cellular Ceramics: Structure, Manufacturing, Properties and Applications, Wiley-VCH, Weinheim Germany (2005) 313.

[3] R. K. Ahluwalia, V. J. Novick, L. Zhang, M. P. Sutaria, J. P. Singh, Performance of a Vacuum Formed Chopped Ceramic Fiber Filter in a Reducing Environment, J. Eng. Gas Turbines and Power 123, 2 (2001) 293.

[4] N. L. Freitas, J. A. S Gonçalves, M. D. M. Innocentini, J. R. Coury, Development of a double-layered ceramic filter for aerosol filtration at high-temperatures: The filter collection efficiency, J. Hazardous Mater. B136 (2006) 747.

[5] R. C. O. Romano, V. C. Pandolfelli, Obtenção e propriedades de cerâmicas porosas pela técnica de incorporação de espuma, Cerâmica 52 (2006) 213.

[6] M. D. M. Innocentini, P. Sepulveda, V. R. Salvini, J. R. Coury, V. C. Pandolfelli, Permeability and structure of cellular ceramics: a comparison between two preparation techniques, J. Am. Ceram. Soc. 81, 12 (1998) 3349.

[7] M. D. M. Innocentini, A. R. F. Pardo, V. R. Salvini, V. C. Pandolfelli, Assessment of Forchheimer's equation to predict the permeability of ceramic foams, J. Am. Ceram. Soc. 82, 7 (1999) 1945.

[8] M. D. M. Innocentini, W. L. Antunes, J. B. Baumgartner, J. P. K. Seville, J. R. Coury, Permeability of ceramic foams 
to gas flow, Adv. Powder Techn. 299 (1999) 19.

[9] M. D. M. Innocentini, V. R. Salvini, A. Macedo, V. C. Pandolfelli, Prediction of ceramic foams permeability using Ergun's equation, Mater. Res. 2, 4 (1999) 283.

[10] M. D. M. Innocentini, V. R. Salvini, J. R. Coury, V. C. Pandolfelli, The permeability of ceramic foams, Am. Ceram. Soc. Bull. 78, 9 (1999) 78.

[11] M. D. M. Innocentini, M. G. Silva, B. A. Menegazzo, V. C. Pandolfelli, Permeability of refractory castables at hightemperatures, J. Am. Ceram. Soc. 84, 3 (2001) 645-647.

[12] D. Hlushkou, U. Tallarek, Transition from creeping via viscous-inertial to turbulent flow in fixed beds, J.
Chromatography A 1126 (2006) 70.

[13] J. P. K. Seville, R. Clift, C. J. Withers, W. Keidel, Rigid ceramic media for filtering hot gases, Filtration and Separation (1989) 265.

[14] E. A. Moreira, M. D. M. Innocentini, J. R. Coury, Permeability of ceramic foams to compressible and incompressible flow, J. Eur. Ceram. Soc. 24 (2004) 3209.

[15] S. Ergun, Flow through packed columns, Chem. Eng. Prog. 48, 2 (1952) 89.

[16] M. D. M. Innocentini, Filtração de gases a altas temperaturas, Tese de doutorado, Universidade Federal de S. Carlos, S. Carlos (1997).

(Rec. 15/04/2008, Ac. 13/06/2008) 